\title{
Cáncer vesical de células escamosas: reporte de un caso y revisión de la bibliografía
}

\section{Squamous cell carcinoma of the bladder: A case report and literature review}

\author{
Luis A Cabrera-Sánchez, ${ }^{1}$ Manuel B Calao-Pérez, ${ }^{1}$ Juan P Acero-Vargas, ${ }^{1}$ Eduardo A González-Murillo, ${ }^{2}$ Juan A \\ Madero-Guerrero, ${ }^{2}$ Anel R Aragón-Tovar ${ }^{1}$
}

\begin{abstract}
Resumen
ANTECEDENTES: El carcinoma de células escamosas es una variedad histológica excepcional del cáncer vesical, pues se estima en $1.6-8 \%$ de los casos.

CASO CLínICO: Mujer de 58 años que inició con cuadro clínico de hematuria macroscópica, formadora de coágulos y anemia. La cistoscopia reportó un tumor vesical de $4 \mathrm{~cm}$ en la pared anterior y fondo vesical. El reporte histopatológico de la resección transuretral de vejiga fue: carcinoma urotelial de alto grado con diferenciación escamosa, invasión a la capa muscular, permeación vascular y perineural. El tratamiento consisitió en cistectomía radical y formación de conducto ileal. Cinco diás después de la cirugía se dio de alta del hospital, sin complicaciones aparentes. El reporte de patología confirmó el diagnóstico de carcinoma escamoso moderadamente diferenciado, con infiltración al tejido perivesical; la inmunohistoquímica resultó positiva para citoqueratina $5 / 6$ y p/63. Con estos resultados se programó a la paciente para recibir tratamiento coadyuvante con radioterapia.
\end{abstract}

CONCLUSIONES: El cáncer vesical es una neoplasia de reporte obligatorio, principalmente sus variedades inusuales (carcinoma de células escamosas), pues contribuye a la estadística nacional y proporciona resultados del tratamiento radical.

PALABRAS CLAVE: Cáncer vesical escamoso; México.

\section{Abstract}

BACKGROUND: Squamous cell carcinoma of the bladder is a rare histologic variety of bladder cancer, accounting for $1.6-8 \%$ of cases.

CLINICAL CASE: A 58-year-old woman presented with clot-forming gross hematuria and anemia. Cystoscopy revealed a $4 \mathrm{~cm}$ tumor on the anterior wall and fundus of the bladder and the histopathologic study from the transurethral resection of the bladder reported high-grade urothelial carcinoma with squamous cell differentiation, invasion of the muscle layer, and vascular and perineural permeation. Treatment was radical cystectomy plus the formation of an ileal pouch. The patient was released on postoperative day 5 with no complications. The pathology study result was moderately differentiated squamous cell carcinoma invading the perivesical tissue, confirming the previous diagnosis. Immunohistochemistry was positive for cytokeratin 5/6 and p/63. The patient was referred for adjuvant radiation therapy.

CONCLUSIONS: The report of unusual types of bladder cancer (squamous cell carcinoma) is vitally important as a contribution to national statistics and to know the results of radical treatment.

KEYWORDS: Squamous cell carcinoma of the bladder; Mexico.

\footnotetext{
${ }^{1}$ Servicio de Urología.

${ }^{2}$ Servicio de Anatomía Patológica.
}

Unidad Médica de Alta Especialidad 25, Centro Médico Nacional del Noreste, Instituto Mexicano del Seguro Social, Monterrey, Nuevo León.

Recibido: junio 2018

Aceptado: octubre 2018

Correspondencia Anel R. Aragón Tovar bercamp9@gmail.com

Este artículo debe citarse como Cabrera-Sánchez LA, Calao-Pérez MB, González-Murillo EA, Madero-Guerrero JA, Aragón-Tovar AR. Cáncer vesical de células escamosas: reporte de un caso y revisión de la bibliografía. Rev Mex Urol. 2018 nov-dic;78(6):440-46. DOI: https://doi.org/10.24245/revmexurol.v78i6.2293 


\section{ANTECEDENTES}

El cáncer vesical es la novena neoplasia maligna más común y la decimotercera causa de muerte por cáncer a nivel mundial. ' Su incidencia varía considerablemente en distintas regiones geográficas; sin embargo, los países industrializados registran las tasas más altas. ${ }^{2}$ De acuerdo con el sexo, los hombres suelen ser los más afectados (relación 3.2:0.9) y la incidencia aumenta con la edad. ${ }^{1}$

El cáncer vesical se origina del epitelio que recubre la superficie interna de la vejiga (urotelio); por tanto, los carcinomas uroteliales representan la mayor parte de los tumores vesicales (9095\%). Las variedades histológicas inusuales suponen menos de $10 \%$ de los $\operatorname{casos}^{3}{ }^{3}$ entre las que destacan los tumores: escamoso o epidermoide, adenocarcinoma y neuroendocrino. Sin embargo, un bajo porcentaje de neoplasias de vejiga corresponde a carcinoma de células escamosas. ${ }^{4}$ En Egipto 10-40\% de los tumores vesicales son escamosos y se asocian con infección por esquistosomiasis, donde esta enfermedad es endémica. $^{5}$

Por corresponder a una estirpe histopatológica poco común, que casi siempre se manifiesta en estadios avanzados, es importante el reporte de este caso.

\section{CASO CLÍNICO}

Paciente femenina de 58 años, ama de casa, con antecedentes personales de hipertensión arterial sistémica, en tratamiento desde hace seis años con losartán; negó tabaquismo, alcoholismo o algún antecedente de exposición a sustancias químicas de riesgo; sin antecedentes de enfermedades urológicas. Inició su padecimiento en enero de 2018 con hematuria macroscópica (formadora de coágulos) y anemia (hemoglobina 8, leucocitos 9.5, plaquetas 394.8 glucosa 64, urea 29.9 creatinina 0.8 , sodio 136 , potasio 4.2 , cloro 114, calcio 9.1, TP 14.8, TTP 23.6, INR 1.1, BT 0.2, TGO 15, TGP 11, FA 81 y DHL 398), por lo que fue enviada al segundo nivel de atención (servicio de Urología). Se realizaron estudios de imagen, cistoscopia y resección transuretral de vejiga, que reportaron un tumor vesical de $4 \mathrm{~cm}$ en la pared anterior y fondo vesical. El reporte histopatológico fue: carcinoma urotelial de alto grado con diferenciación escamosa, invasión a la capa muscular, permeación vascular y perineural.

Con estos hallazgos fue enviada al servicio de Urología del Centro Médico Nacional del Noreste para tratamiento radical. Se realizó protocolo preoperatorio, estudios de imagen (TAC abdominopélvica simple y contrastada (Figura 1) y revisión de portaobjetos, que confirmaron el diagnóstico histopatológico inicial. Se estadificó a la paciente en T2b NOMO EC II. El 15 de marzo de 2018 se efectuó cistectomía radical, linfadenectomía pélvica bilateral y derivación urinaria uretero-ileal tipo Bricker, con engrapadora lineal para la fabricación del conducto ileal y uso de tutores ureterales retirados al séptimo día posquirúrgico. Se prescribió enoxaparina después de 12 h posquirúrgicas, con deambulación temprana e inicio de dieta enteral progresiva al tercer día, sin

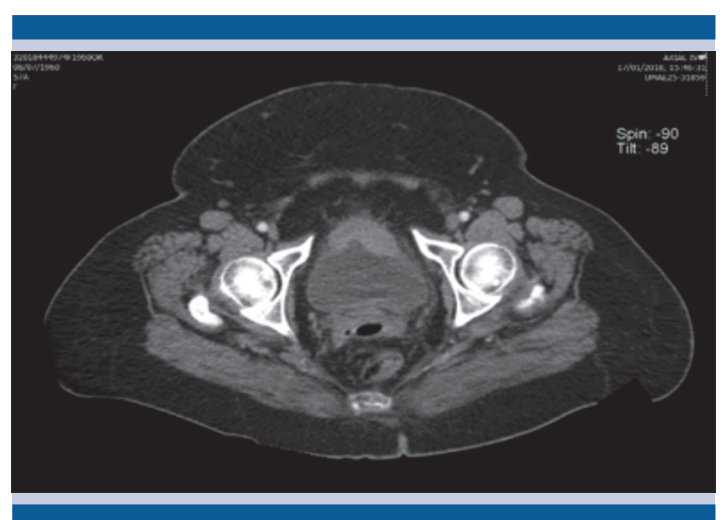

Figura 1. Tomografía contrastada que muestra un tumor vesical en la cara anterior e incremento en la densidad de la grasa perivesical. 


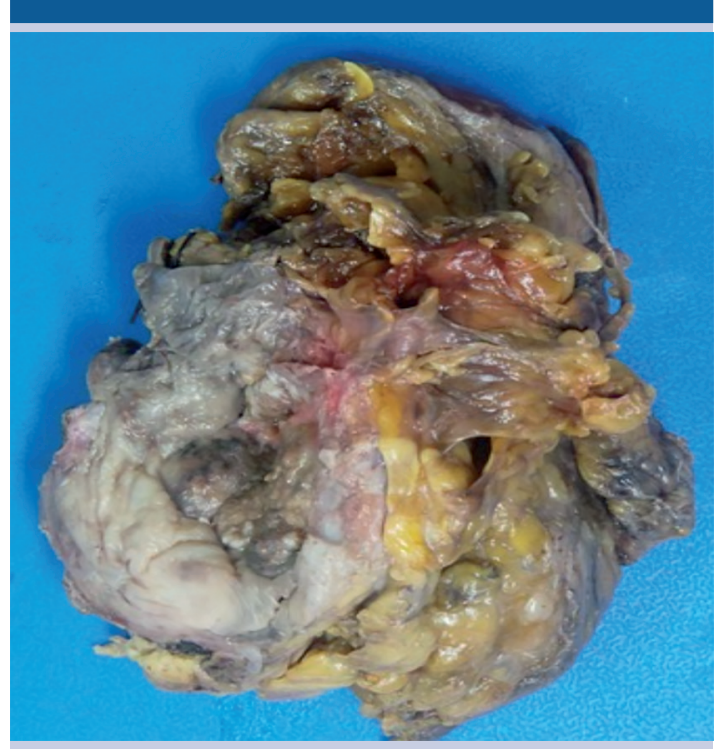

Figura 2. Vejiga con tumor exofítico, de $4 \times 3 \times 2 \mathrm{~cm}$, localizado en la pared antero-lateral derecha, de color blanquecino, congestivo, de consistencia semifirme.

requerimiento de sonda nasográstrica. Al quinto día del procedimiento quirúrgico se otorgó el alta hospitalaria, con seguimiento en consulta externa, sin complicaciones posquirúrgicas.

Los hallazgos patológicos fueron: vejiga con tumor de $4 \mathrm{~cm}$ en su diámetro mayor, localizada en la pared anterior y fondo vesical (Figura 2), con resultado histopatológico de carcinoma escamoso moderadamente diferenciado queratinizante, que infiltraba hasta el tejido adiposo perivesical, con invasión angiolinfática y perineural (Figuras 3 y 4). Estadificación: pT3bN2M0 EC IIIB, útero y anexos libres de actividad tumoral; linfadenectomía pélvica derecha e izquierda con ganglios de $5 \mathrm{~cm}$, positiva para carcinoma epidermoide (3/3) y $(2 / 2)$, respectivamente; e inmunohistoquímica positiva para citoqueratinas $5 / 6$ y $p / 63$. Figuras 5 y 6

De acuerdo con el resultado histopatológico, la paciente fue enviada para iniciar tratamiento coadyuvante con radioterapia en el servicio de Oncología de nuestro centro.

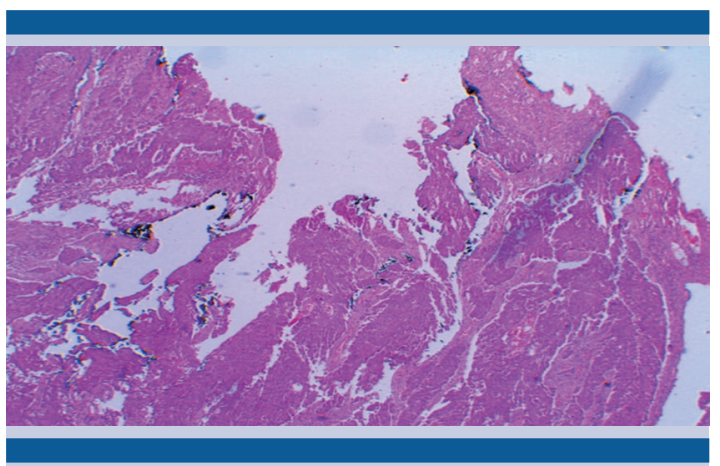

Figura 3. Histología (40X) que evidencia células epiteliales escamosas neoplásicas, moderadamente diferenciadas, con zonas de queratinización.

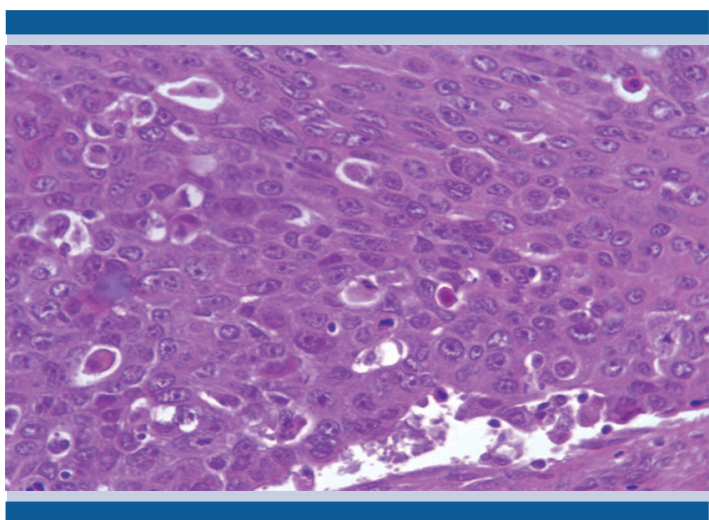

Figura 4. Histología (100X) con hallazgos de carcinoma escamoso moderadamente diferenciado, queratinizante, con invasión angiolinfática y perineural.

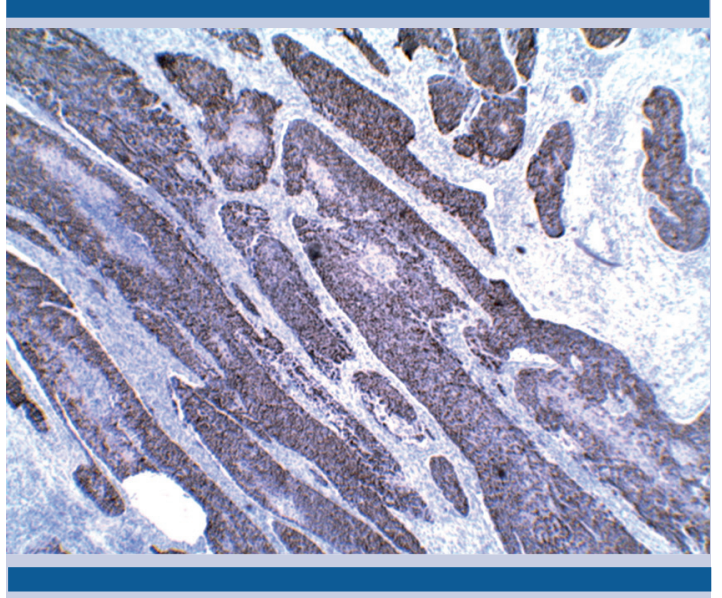

Figura 5. Inmunohistoquímica con positividad citoplasmática para citoqueratina $5 / 6$ en las células neoplásicas. 


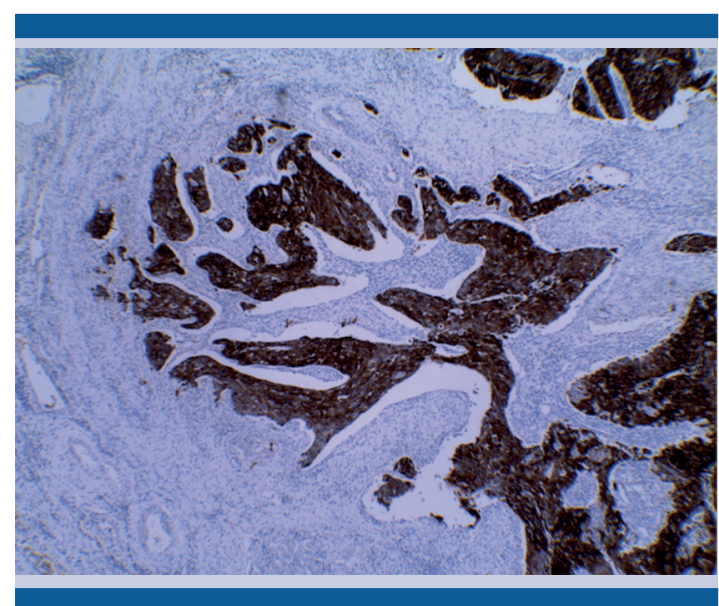

Figura 6. Inmunohistoquímica con positividad nuclear para p/63 en las células neoplásicas.

\section{DISCUSIÓN}

El cáncer vesical es un problema de salud relevante en todo el mundo, representa la novena neoplasia más común en hombres y mujeres, y supone una causa importante de mortalidad específica por cáncer. ${ }^{6}$

Entre los múltiples tipos histológicos del cáncer vesical, el urotelial se asocia con una prevalencia aproximada de $90 \%$, mientras que el carcinoma de células escamosas representa 1.6 a $8 \%$ de todos los casos. ${ }^{7-10}$ Este último suele manifestarse en pacientes de 70.9 años de edad (49-88 años). ${ }^{7-9}$ En México se ha estimado en $2.2 \%$ de las neoplasias malignas y representa la decimoquinta causa de mortalidad por cáncer. ${ }^{11}$

Existen pocos casos reportados de carcinoma de células escamosas en la bibliografía mexicana. Es evidente la subestimación de los casos, debido a la falta de publicación. El caso aquí expuesto supone el cuarto publicado en las revistas nacionales y representa el primero de estirpe escamosa en 20 años de vigilancia de cáncer vesical en nuestra institución.

\section{Factores de riesgo}

El tabaquismo se ha reportado como el principal factor de riesgo para cáncer vesical (tanto urotelial, como tumores inusuales). Entre los factores de riesgo para carcinoma de células escamosas se encuentran: inflamación-irritación crónica, cateterización a largo plazo, vejiga neurogénica, cálculos en la vía urinaria inferior e infección crónica por Schistosoma haematobium, especialmente en áreas endémicas. ${ }^{10,12,13}$ La irritación vesical estimula la producción de citocinas que favorecen la proliferación y migración celular, angiogénesis e inhibición de la apoptosis, lo que resulta en metaplasia escamosa, displasia $y$, finalmente, un tumor maligno. ${ }^{14}$

Aunque la infección de vías urinarias inferiores por gérmenes gramnegativos representa un hallazgo relativamente frecuente, la paciente de este estudio no tenía antecedentes de tabaquismo, infección urinaria, litiasis vesical ni vejiga neurogénica.

\section{Diagnóstico}

Las manifestaciones clínicas en pacientes con carcinoma de células escamosas son indistinguibles de quienes tienen cáncer vesical urotelial. La mayoría inicia con hematuria macroscópica y síntomas en la vía urinaria inferior (disuria, urgencia y frecuencia). ${ }^{10}$ El dolor pélvico y lumbar, y la hidronefrosis son sugerentes de enfermedad avanzada. Algunos estudios indican que 93\% de los pacientes puede padecer infección de vías urinarias al momento del diagnóstico. ${ }^{15}$ Los estudios de imagen evidencian hallazgos similares en pacientes con cáncer vesical urotelial y carcinoma de células escamosas.

La expresión del carcinoma de células escamosas superficial es excepcional. Los estadios Ta y T1 son poco frecuentes; sin embargo, T3 representa la etapa más común (60\%) como sucedió en nuestro caso. ${ }^{16}$ 
El carcinoma de células escamosas es una neoplasia epitelial con características histológicas propias, como: perlas escamosas, puentes intercelulares y gránulos de queratohialina. ${ }^{17}$ Las características del cáncer vesical urotelial excluyen el diagnóstico de carcinoma de células escamosas puro; por tanto, debe definirse como cáncer vesical urotelial mixto o con diferenciación escamosa. En el caso aquí reportado, la pieza histopatológica definitiva (después del tratamiento quirúrgico radical) mostró un carcinoma de células escamosas puro, a pesar de los hallazgos previos en la resección transuretral de vejiga (patrón mixto).

En cuanto a las pruebas de inmunohistoquímica, no existen diferencias entre el carcinoma de células escamosas vesical y el localizado en otras zonas genitourinarias. El carcinoma de células escamosas muestra un patrón de citoqueratinas de alto peso molecular y prequeratinas, a diferencia del cáncer vesical urotelial, que tiene citoqueratinas de bajo peso molecular. ${ }^{18}$

Este tipo de tumores representa un reto diagnóstico para el patólogo y terapéutico para el urólogo, pues son neoplasias poco comunes. En algunos casos se dificulta su clasificación anatomopatológica y su comportamiento clínico es diferente, por lo que se requiere un tratamiento individualizado. ${ }^{8}$

\section{Tratamiento}

La bibliografía médica reporta poca experiencia en el tratamiento de pacientes con cáncer vesical escamoso; sin embargo, la mayoría se beneficia del diagnóstico oportuno y cirugía radical. Estudios recientes demuestran resultados satisfactorios con la cistectomía radical y linfadenectomía pélvica bilateral, con supervivencia a 5 años de $65-75 \%$ de los casos en estadio T1-T2 y de $15-35 \%$ en T3-T4. ${ }^{19}$
Los límites de la disección linfática de nuestra paciente fueron (linfandenectomía estándar): el nervio genitofemoral y la pared pélvica lateral; la vena circunfleja y el ganglio de Cloquet distalmente; la fosa obturadora y los vasos iliacos internos posteriormente, y el tejido medial a estos vasos, incluida la región presacra, proximal al tercio superior de los vasos iliacos comunes. ${ }^{20,21}$

Diferentes estudios han demostrado que el número de ganglios linfáticos resecados representa un factor pronóstico en pacientes con cáncer de vejiga. La variación de 8 a 56 ganglios removidos es una variabilidad interindividuo. El principal objetivo es obtener más de 20 ganglios por paciente. ${ }^{21,22}$

La densidad ganglionar (relación entre los ganglios positivos y el total de ganglios removidos) se considera otro factor pronóstico importante (se expresa en porcentaje). Los pacientes con $\leq$ $20 v s \geq 20 \%$ tienen probabilidad de 43 y $17 \%$ de supervivencia libre de enfermedad a 10 años, respectivamente. ${ }^{22}$

El estudio de Kassouf y su grupo, efectuado en 27 pacientes con cáncer vesical de células escamosas, atendidos en el MD Anderson Cancer Center (1988-2003), reportó que la cistectomía radical es el tratamiento de elección en sujetos con cáncer local. ${ }^{23}$

La base de datos del SEER (Surveillance, Epidemiology, and End Results Program), de 1973 a 2013, ofrece una visión general de las tendencias epidemiológicas y de tratamiento para el cáncer de células escamosas. De acuerdo con el análisis de ese periodo, se registraron 5018 pacientes con este tipo de cáncer (mediana de edad de 70-75 años) y de éstos 2585 fueron varones; se realizó cistectomía radical en 2457 casos y al evaluar la supervivencia global en sujetos a quienes se efectuó cirugía radical, resultó significativamente superior ( $p<0.0001$ ) versus radioterapia como único tratamiento. ${ }^{24}$ 


\section{Pronóstico}

Los factores pronóstico en pacientes con carcinoma de células escamosas incluyen: estadio patológico, invasión linfovascular y a ganglios linfáticos pélvicos. El $90 \%$ de la mortalidad se debe a recurrencia pélvica local. La metástasis a distancia es poco frecuente, con incidencia menor de $10 \% .{ }^{25}$

\section{CONCLUSIONES}

De acuerdo con nuestra revisión de la bibliografía internacional y los pocos casos publicados en México, el tratamiento de pacientes con carcinoma de células escamosas no metastásico consiste en cistectomía radical, pues proporciona mayor supervivencia global, comparada con la radioterapia. El cáncer vesical sigue siendo una neoplasia de reporte obligatorio, principalmente sus variedades inusuales (carcinoma de células escamosas), pues contribuye con la estadística nacional y proporciona resultados del tratamiento radical.

\section{REFERENCIAS}

1. Ferlay J, et al. Cancer incidence and mortality worldwide: sources, methods and major patterns in GLOBOCAN 2012. Int J Cancer 2015;136(5):E359-E386. DOI: 10.1002/ijc.29210

2. Sanli O, et al. Bladder cancer. Nat Rev Dis Primers 2017;3:17022. DOI: 10.1038/nrdp.2017.22

3. Willis D, Kamat AM. Nonurothelial bladder cancer and rare variant histologies. Hematol Oncol Clin North Am 2015;29(2):237-252. DOI: 10.1016/j.hoc.2014.10.011

4. Shariat SF, et al. Outcomes of radical cystectomy for transitional cell carcinoma of the bladder: a contemporary series from the Bladder Cancer Research Consortium. J Urol 2006;176(6 Pt 1):2414-22. DOI: 10.1016/j.juro.2006.08.004

5. Parkin DM. The global burden of urinary bladder cancer. Scand J Urol Nephrol 2008;(218):12-20.

6. Torre LA, et al. Global cancer statistics, 2012. CA Cancer J Clin 2015;65:87-108. DOI: 10.3322/caac.21262

7. Ricos-Torrent JV y cols. El carcinoma epidermoide de vejiga un tumor poco común. Arch Esp Urol 1989;42:205.

8. Queipo-Zaragoza JA, et al. Unusual bladder tumors: primary epidermoid carcinoma, adenocarcinoma, and sarcoma. Clinical behavior. Our experience. Actas Urol Esp 2003;27(2):123-131.

9. Budia-Alba A, et al. Comparative study epidermoid carcinoma of the bladder and transitional cell carcinoma with squamous or mixed differentiated foci. Actas Urol Esp 1999;23(2):111-8.

10. Shokeir AA. Squamous cell carcinoma of the bladder: pathology, diagnosis and treatment. BJU Int 2004;93(2):216-20.

11. Sedano-Basilio JE, et al. Epidemiología de los tumores genitourinarios en una década. Rev Mex Urol 2016;76(3):131140.

12. Ehdaie B, et al. Comparative outcomes of pure squamous cell carcinoma and urothelial carcinoma with squamous differentiation in patients treated with radical cystectomy. J Urol 2012;187:74-9. DOI: 10.1016/j.juro.2011.09.056

13. Mostafa $\mathrm{MH}$, et al. Relationship between Schistosomiasis and bladder cancer. Clin Microbiol Rev 1999;12:97-111.

14. Youssef $\mathrm{R}$, et al. Bilharzial vs non-bilharzial related bladder cancer: pathological characteristics and value of cyclooxygenase-2 expression. BJU Int 2011;108:31-7. DOI: 10.1111/j.1464-410X.2010.09854.x

15. Hicks RM, et al. Association of bacteriuria and urinary nitrosamine formation with Schistosoma haematobium infection in the Qalyub area of Egypt. Trans R Soc Trop Med Hyg 1982;76(4):519-27.

16. Lagwinski $\mathrm{N}$, et al. Squamous cell carcinoma of the bladder: a clinicopathologic analysis of 45 cases. Am J Surg Pathol 2007;31:1777-87. DOI: 10.1097/PAS.0b013e31805c9cd9

17. Manunta A, et al. Non-transitional cell bladder carcinomas. BJU Int 2005;95:497-502. DOI: 10.1111/j.1464410X.2005.05327.x

18. González-Resina R, et al. Carcinoma epidermoide vesical. Revisión de nuestra serie. Arch Esp Urol 2006;59:8,785790.

19. El-Sabaie M, et al. Squamous cell carcinoma of the bilharzial and non-bilharzial urinary bladder: a review of etiological features, natural history and management. Int J Clin Oncol 2005;10(1):20-25. DOI: 10.1007/s10147-004-0457-6

20. Abol-Enein $\mathrm{H}$, et al. Does the extent of lymphadenectomy in radical cystectomy for bladder cancer influence diseasefree survival? A prospective single-center Study. Eur Urol 2011;60(3):572-577. DOI: 10.1016/j.eururo.2011.05.062

21. Karl A, et al. The impact of lymphadenectomy and lymph node metastasis on the outcomes of radical cystectomy of bladder cancer. Eur Urol 2009;55(4):826-835. DOI: 10.1016/j.eururo.2009.01.004

22. Zehnder $P$, et al. Super extended versus extended pelvich node dissection in patients undergoing radical cystectomy for bladder cancer: A comparative study. J Urol 2011;186(4):1261-268. DOI: 10.1016/j.juro.2011.06.004 
23. Kassouf W, et al. Outcome and patterns of recurrence of nonbilharzial pure squamous cell carcinoma of the bladder: a contemporary review. Of The University of Texas M D Anderson Cancer Center experience. Cancer 2007;110:764-9. DOI: 10.1002/cncr.22853

24. Lenis AT, et al. Association between number of endoscopic resections and utilization of bacillus Calmette-Guérin therapy for patients with high-grade, non-muscle-invasive bladder cancer. Clin Genitourin Cancer 2017;15(1):e25-31. DOI: 10.1016/j.clgc.2016.06.014

25. Abol-Enein $\mathrm{H}$, et al. Nonurothelial cancer of the bladder. Urology 2007;69:93-104. DOI: 10.1016/j.urology.2006.08.1107

\section{AVISO IMPORTANTE}

La Revista Mexicana de Urología se convierte en una publicación solo digital, con todas las ventajas que los medios y dispositivos electrónicos ofrecen. Usted podrá revisar la información mediante el sitio web (www.revistamexicanadeurologia.org.mx) o descargando la app para Android o iPhone.

Para consultar el texto completo de los artículos deberá registrarse por una sola vez con su correo electrónico, crear una contraseña, indicar su nombre, apellidos y especialidad.

Esta información es indispensable para saber qué consulta y cuáles son sus intereses, y poder en el futuro inmediato satisfacer sus necesidades de información. 